\title{
Observations of vortex-like structure in the cusp-magnetosheath region during northward IMF orientation
}

\author{
O. Tkachenko ${ }^{1}$, J. Šafránková ${ }^{1}$, Z. Němeček ${ }^{1}, J_{\text {S. Šmůnek }}{ }^{2}$, and L. Přech ${ }^{1}$ \\ ${ }^{1}$ Charles University, Faculty of Mathematics and Physics, V Holesovickach 2, 18000 Praha 8, Czech Republic \\ ${ }^{2}$ Institute of Atmospheric Physics, Czech Academy of Science, Boční 1405, Praha 4, Czech Republic
}

Received: 4 April 2008 - Revised: 27 August 2008 - Accepted: 11 September 2008 - Published: 22 October 2008

\begin{abstract}
Two-point observations made by the Interball spacecraft pair unveiled a presence of a large vortex-like structure filled by slow and heated plasma in the outer cusp during periods of the positive $B_{Z}$ interplanetary magnetic field (IMF) component. We have observed the rotation of the magnetic field and the decrease of its magnitude connected with the presence of a hot plasma population inside the vortex. The structure starts with a clear reversal of the plasma flow caused by reconnection tailward of the cusp that turns a part of the magnetosheath plasma into the cusp. We compare the observations of two spacecraft in detail, discuss the differences between them and suggest the way of a formation such structure. Moreover, the statistical results have confirmed that this phenomenon is probably a regular feature of the high-altitude cusp, however, this relatively small structure cannot be observed too often. The alternative explanation of presented observations as a flapping of the magnetopause is taken into account and discussed.
\end{abstract}

Keywords. Magnetospheric physics (Magnetopause, cusp, and boundary layers; Solar wind-magnetosphere interactions) - Space plasma physics (Magnetic reconnection)

\section{Introduction}

Classical theory of interaction between the solar wind and the magnetosphere predicts the magnetopause to be an impenetrable boundary separating cold dense plasmas on magnetosheath magnetic field lines from hot tenuous plasmas on magnetospheric field lines. Observations indicate that a boundary layer of magnetosheath-like plasmas can be found inside all regions of the magnetopause, including the nightside equatorial magnetopause (Hones et al., 1972), the low-latitude dayside magnetopause (Eastman et al., 1976; Haerendel et al., 1978), and the high-latitude magnetopause

Correspondence to: J. Šafránková

(jana.safrankova@mff.cuni.cz)
(Rosenbauer et al., 1975; Paschmann et al., 1976). The boundary layer is divided into the low-latitude boundary layer, the entry layer near the polar cusps, and the plasma mantle along the high-latitude magnetotail.

The interconnection of the interplanetary magnetic field (IMF) with the Earth's magnetic field has a dominant effect on the magnetosphere and ionosphere; it is believed to be the main mechanism for both plasma and energy inputs from the solar wind. Several mechanisms of magnetosheath plasma entry have been suggested. Present observational facts are generally consistent with magnetic reconnection being a dominant source of the cusp plasma, whereas other mechanisms can contribute to the cusp population under specific circumstances. As noted Sibeck et al. (1999), a unique feature of reconnection is that it requires the relevant physical processes to take place only in a narrow diffusion region, while its consequences are global: Once the interplanetary and magnetospheric lines become interconnected, they remain connected while being convected with the solar wind, and plasma continues to enter the magnetosphere. This is in contrast to all other mechanisms that operate only locally.

Concerning the location of the reconnection site, Crooker (1979) and Luhmann et al. (1984) suggested that reconnection occurs when antiparallel magnetosheath and magnetospheric magnetic field lines come into contact. Reconnection on the dayside equatorial magnetopause can occur when IMF points southward (Dungey, 1961, 1963; Reiff et al., 1977; Reiff, 1984) and with open lobe magnetic field lines on the polar magnetopause when IMF has a northward component (Burch et al., 1980; Song and Russell, 1992). In this case, reconnection occurs at high-latitudes poleward of the Earth's magnetic cusps (e.g. Gosling et al., 1991; Kessel et al., 1996; Russell et al., 1998; Šafránková et al., 1998; Fuselier et al., 2000; Onsager et al., 2001; Měrka et al., 2002; Cargill et al., 2005; Escoubet et al., 2008).

Published by Copernicus Publications on behalf of the European Geosciences Union. 


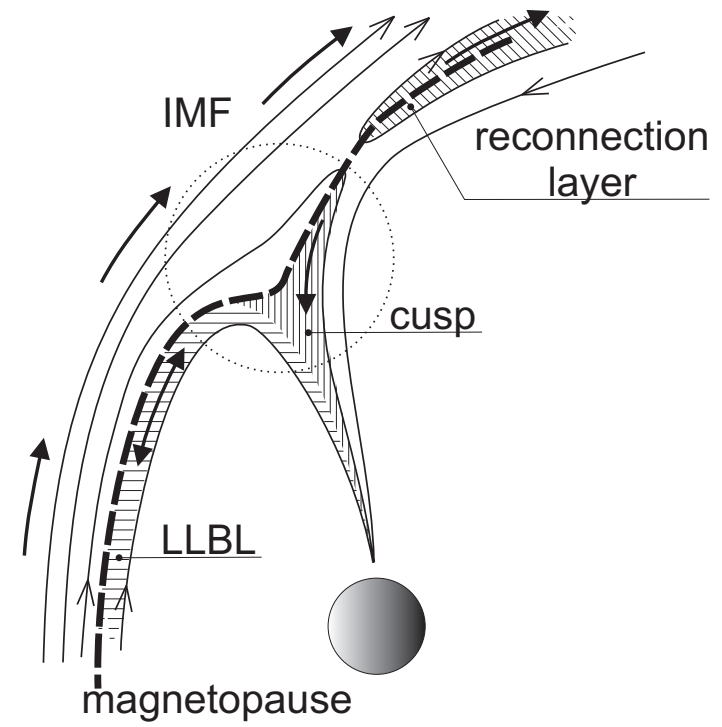

Fig. 1. The cusp configuration during a prolonged interval of northward pointing IMF. The light lines represent IMF and Earth's magnetic field lines; the heavy arrows show the dominating flow directions in the cusp and adjacent regions. The dotted circle shows the region under study.

When reconnection takes place equatorward of the cusp, the convective flow is antisunward and magnetospheric open field lines are pulled poleward through the cusps. The whole dayside magnetopause is open and should be probably placed through kinks of the magnetic field lines that are in a permanent motion tailward. Magnetic field minima at the cusps lead to indentation of the magnetopause. Such indentation results from the pressure balance at the magnetopause (e.g. Sotirelis and Meng, 1999) because the magnetic field exhibits minima at these regions (e.g., Cargill et al., 2004). The indentation was predicted by Spreiter and Briggs (1962) and later detected by the different spacecraft, HEOS2, ISEE, Hawkeye, Interball-1, Magion-4 (e.g. Paschmann et al., 1975; Rosenbauer et al., 1975; Petrinec and Russell, 1995; Němeček et al., 2000). Recently, Šafránková et al. (2002a) suggested and Šafránková et al. (2005) confirmed the presence of a magnetopause indentation in the cusp region. Zhang et al. (2007) have analyzed Cluster observations, both statistically and for individual crossing cases, and concluded that the boundary between the magnetosheath and cusp seems to be indented in the $X-Y$ plane and it is less clearly indented in the $X-Z$ plane. This indentation can slow down the magnetosheath flow (Němeček et al., 2007). Lavraud et al. (2004a,b) broadly discuss this problem and suggest that the indented magnetopause is not the magnetopause itself but the inner boundary of the exterior cusp. However, regardless of which of these mechanisms dominate, a careful analysis of Cluster observations made by Cargill et al. (2005) has undoubtedly shown the sub-alfvénic flow in the questioned region.
On the other hand, when the reconnection site is located poleward of the cusp, the resulting convective flow is sunward and an open field line is pushed equatorward through the cusp in this case (Lavraud et al., 2002). The convective flow is slow because it opposes the fast magnetosheath bulk flow. The subsolar magnetopause position and the cusp latitude are not sensitive to reconnection rate variations during northward IMF (e.g. Newell et al., 1989; Palmroth et al., 2001; Šafránková et al., 2002b).

The present understanding of the structure of the cuspmagnetosheath interface formed by northward pointing IMF is illustrated in Fig. 1. The magnetic field lines reconnected at one hemisphere can re-reconnect tailward of the conjugated cusp and close the magnetosheath plasma on dayside magnetospheric field lines. On the other hand, the tail part of the reconnected lobe line is decoupled from the Earth and becomes an IMF line. This process leads to the creation of a layer of tailward flowing plasma adjacent to the night-side high-latitude magnetopause. This layer (called reconnection layer in Šafránková et al., 1998; Longmore et al., 2005) has similar properties as the plasma mantle that can be found at the same location during southward IMF intervals but there are two principal differences: the reconnection layer is outside the magnetopause and it is supplied with the magnetosheath plasma directly.

When dayside (LLBL) lines are closed the particles are bouncing and they convect with the magnetic field to the nightside. The presence of horizontal IMF components can prevent re-reconnection and the dayside LLBL is on open field lines in such a case. A broad region equatorward of the entry point is filled with the heated plasma due to its convection equatorward, magnetic mirroring and turbulence excited by the counterstreaming flows.

The magnetopause is indented due to the depressed magnetic field in this region. Consequently, there is a volume above the magnetopause (encircled in Fig. 1) that would exhibit a different plasma behavior than the free-flow magnetosheath. Sotirelis and Meng (1999) suggested a secondary stagnation point in this region when the magnetic dipole is tilted toward the Sun in a particular hemisphere and Haerendel et al. (1978) argued that the whole volume would be filled with a stagnant plasma. The knowledge of the plasma parameters in this volume is very important because the plasma there supplies the cusp during periods of the northward IMF. Unfortunately, this region is visited by the spacecraft rather rarely and thus there are only a few studies dealing with the plasma flow and magnetic field just above the cusp (e.g. Dunlop et al., 2002; Němeček et al., 2003; Bogdanova et al., 2005; Zong et al., 2005; Dunlop et al., 2005; Panov et al., 2008). 


\section{Vortex-like structure observations on different scales}

Different types of self-organized structures, vortices, were frequently observed in a variety of environments including the heliosphere (Burlaga, 1990; Zhou et al., 2004) and the Earth's ionosphere and magnetosphere (e.g. Hones et al., 1981; Savin et al., 1998, 2002, 2004).

Savin et al. (1998) discussed non-linear vortex waves on medium time-scales in the so-called outer cusp throat and outside the magnetopause in the turbulent boundary layers. The authors supposed that their vortices form due to tailward reconnection and propagate upstream of the outer throat deflecting the magnetosheath flow at the outer boundary. In a comprehensive study, Savin et al. (2004) presented that three types of populating processes should be in operation at the magnetosheath-cusp interface: (1) large-scale $\left(\sim\right.$ few $\left.R_{E}\right)$ antiparallel merging at the sites remote of the cusp, mediumscale (few thousand of $\mathrm{km}$ ) local merging at the turbulent layer, and (3) small-scale (few hundred of $\mathrm{km}$ ) bursty reconnection of fluctuating magnetic fields.

Alexandrova et al. (2006) have reported space localized coherent magnetic structures in the form of Alfvén vortices in the magnetosheath downstream of a quasi-perpendicular bow shock. The authors discuss the possible origins of these vortices and suggest a strong turbulence or the filamentation instability of an Alfvén wave.

In the cusp region, Sundkvist et al. (2005) have studied short-scale drift-kinetic Alfvén vortices. Their dynamic structures observed by the Cluster spacecraft have a characteristic scale of the order of the ion gyroradius $(\sim 25 \mathrm{~km})$ and correspond to an isolated vortex model (Shukla et al., 1986; Liu and Horton, 1986) for conditions applicable to the cusp plasma.

These structures contrast to a recent observation (Hasegawa et al., 2006) of larger Kelvin-Helmholtz vortices with a typical diameter of $\sim 6 R_{E}$. These vortex-like structures were reported usually in the LLBL and they are associated with the Kelvin-Helmholtz instability at the magnetospheric flank (e.g. Hasegawa et al., 2004, 2006; Nykyri et al., 2006; Volwerk et al., 2007, 2008). Hasegawa et al. $(2004,2006)$ demonstrated the non-linear rolled-up nature of such vortices. They proposed the Kelvin-Helmholtz instability as a candidate of local entry mechanisms of a solar wind transport during northward IMF orientation in the absence of active reconnection at low-latitudes. Recent numerical simulation models (e.g. Otto and Fairfield, 2000; Nykyri and Otto, 2001) suggest that fast plasma transport across the magnetopause can be mediated by the KelvinHelmholtz instability only when it is growing sufficiently to form rolled-up vortices that can mix plasmas from both sides of the magnetopause. In these models, the collapse of or reconnection within such a vortex is responsible for the plasma transport.

From this short survey it follows that the occurrence of vortices is a very common feature of the solar wind- magnetosphere interaction. Their sources are connected with a turbulent environment, for example with a non-linear growth of either Alfvén waves or Kelvin-Helmholtz instability. During a period of positive IMF $B_{Z}$, Šafránková et al. (2002b) and Měrka et al. (2005) have discussed a vortexlike cavity filled by slow-moving heated plasma inside the boundary layer in the outer cusp. However, both papers leave this interesting event without an explanation. The present paper continues this study; discusses this kind of a vortexlike structure created by a turbulent plasma flow around the magnetopause indentation above the cusp and, using the data from Interball-1 and Magion-4, finds necessary conditions and a possible mechanisms to creation of such structures.

\section{Upstream conditions and overview of the event}

The study is based on plasma flow and magnetic field patterns observed by the Interball-1 and Magion- 4 spacecraft that moved along the same orbit. The inclination of the orbits was $68^{\circ}$, thus the spacecraft often scanned the vertical profile of the cusp region from middle altitudes toward the magnetopause (Sandahl et al., 1997). Both satellites were spin stabilized with the spin axis pointing toward the Sun and both were equipped with magnetometers, ion flow measurements, and ion spectrometers (e.g. Němeček et al., 1997; Šafránková et al., 1997; Klimov et al., 1997; Yermolaev et al., 1997). The entry windows of each spectrometer created a fan which covered nearly the whole $4 \pi$ of a spatial angle during one satellite revolution. Interball-1 rotated with the period of about $\sim 120 \mathrm{~s}$ and the spin period of Magion- 4 was $\sim 60 \mathrm{~s}$ in the analyzed case. The plasma spectrometers used the spacecraft spin for angular scanning of the velocity distribution and thus the basic plasma moments are available with the spin time resolution. The magnetic field vector is available with a $1 \mathrm{~s}$ resolution.

A vortex-like structure (Měrka et al., 2005) was observed by both spacecraft on 5 June 1996 between 18:20 and 19:00 UT. The spacecraft moved outward from the magnetosphere and crossed the magnetopause at $(2.9 ;-2.2 ; 8.7) R_{E}$. The "temporal separation" of the spacecraft was $\approx 540 \mathrm{~s}$, Magion-4 being ahead of Interball-1. The spatial separation slightly varied during the interval under study and was $(0.22 ;-0.7 ; 0.13) R_{E}$ at $18: 30 \mathrm{UT}$.

The locations of both spacecraft with respect to the magnetopause position is shown in Fig. 2. The full line shows the magnetopause position according to the Petrinec and Russell (1996) model and the dashed line uses the correction suggested by Šafránková et al. (2005) for the position of the high-latitude magnetopause near the cusp. We note that both spacecraft cross the magnetopause inside the indentation caused by the depression of the magnetic field in the cusp region; first by Magion 4 at 18:41 UT and then by Interball-1 at 18:51 UT. 


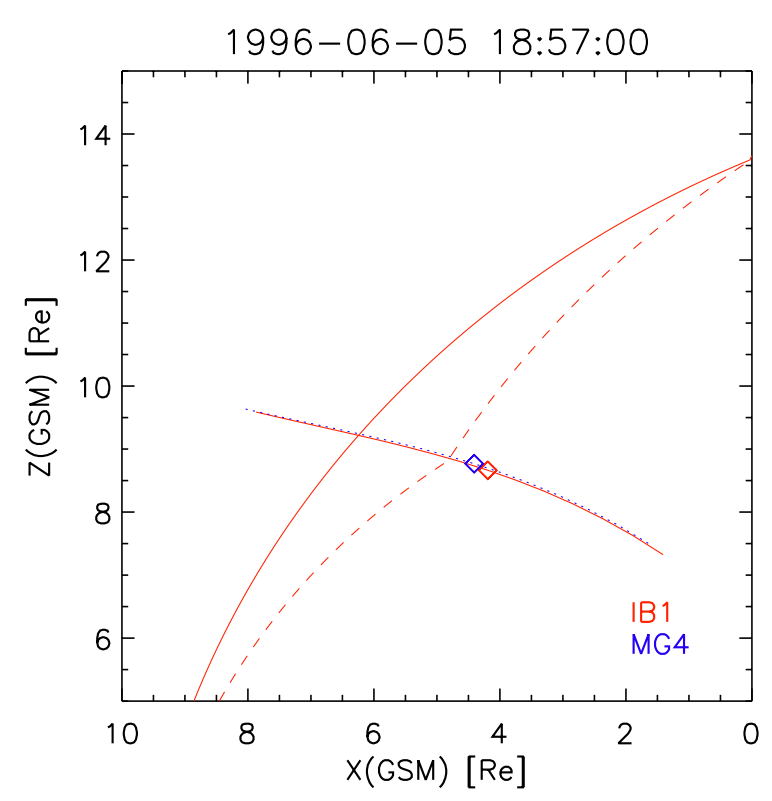

Fig. 2. The locations of both Interball spacecraft inside the cusp region near the magnetopause. The full line displays the magnetopause location according to the Petrinec and Russell (1996) model and the dashed line shows the corrected magnetopause location (according to Šafránková et al., 2005).

Solar wind parameters, IMF strength and components monitored far upstream by Wind $\left(181,0 ; 6,3 ;-13,2 R_{E}\right.$ in GSM coordinates) and IMP 8 (close to the bow shock) are displayed in Fig. 3 and show relatively stable conditions (solar wind dynamic pressure $\approx 2.7 \mathrm{nPa}$ with the solar wind speed $\approx v_{S W}=350 \mathrm{~km} / \mathrm{s}$ and density varying between 9 and $11 \mathrm{~cm}^{-3}$ ). The best agreement of the IMF changes with those observed by IMP 8 (located in $(19,7 ;-25,3 ; 23,5) R_{E}$ in GSE coordinates) was achieved for the time lag of $38 \mathrm{~min}$ that is lower than the lag computed from the spacecraft separation and solar wind velocity ( $49 \mathrm{~min})$. For this reason, we use the propagation speed derived from this comparison and apply it for determination of the time lag of both Wind and IMP 8 data in Fig. 3.

For our study, it is important that the IMF points generally tailward and northward and the IMF $B_{Y}$ component is negative or nearly zero during the investigated interval. A small value of IMF $B_{Y}$ makes the analysis easier because a large $B_{Y}$ value shifts the reconnection site dawnward/duskward of the cusp proper and the possible inflow from the conjugated hemisphere should be considered (e.g. Němeček et al., 2003).

\section{Magion-4 and Interball-1 measurements}

The studied structure was first observed by Magion- 4 in the time interval from 18:41:00 to 18:45:30 UT and can be clearly identified in the changes of the magnetic field di-

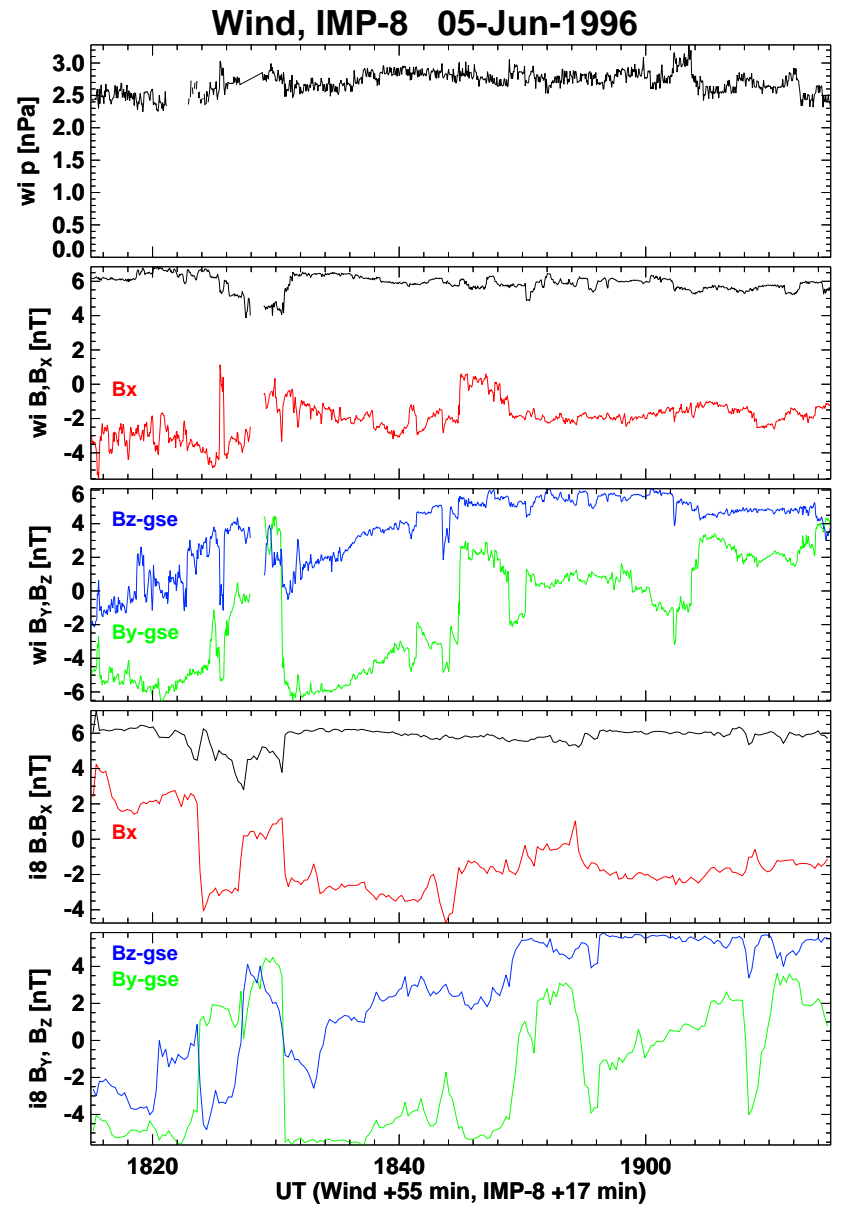

Fig. 3. Overview of solar wind and IMF WIND and IMP 8 measurements during the 5 June 1996 event. From top to bottom: WIND solar wind dynamic pressure; WIND IMF magnitude and three components (two panels); and IMP 8 IMF magnitude and three components (two panels). The Wind and IMP 8 data are propagated to the Interball-1 location.

rections (Fig. 4) that exhibit a complicated rotation during the crossing of a thick boundary layer. At the beginning of the interval (until 18:41:30 UT), the measured magnetic field has the magnetospheric orientation and its orientation is magnetosheath-like at the end of the event (18:45:30 UT). The ion energy spectra in the middle panels of Fig. 5 were measured in antisunward $(E i 0)$, perpendicular $(E i 90)$ and sunward (Ei 180) directions. The black lines inside the panels indicate a pitch angle of a particular analyzer. The detail inspection of the spectrograms allows us to divide a whole interval into several parts that are distinguished by the lines and numbers in Fig. 4. The properties of the plasma and magnetic field inside the numbered intervals can be described as follows:

1. Magnetospheric magnetic field and upgoing ions of magnetosheath energies are signatures of the plasma 


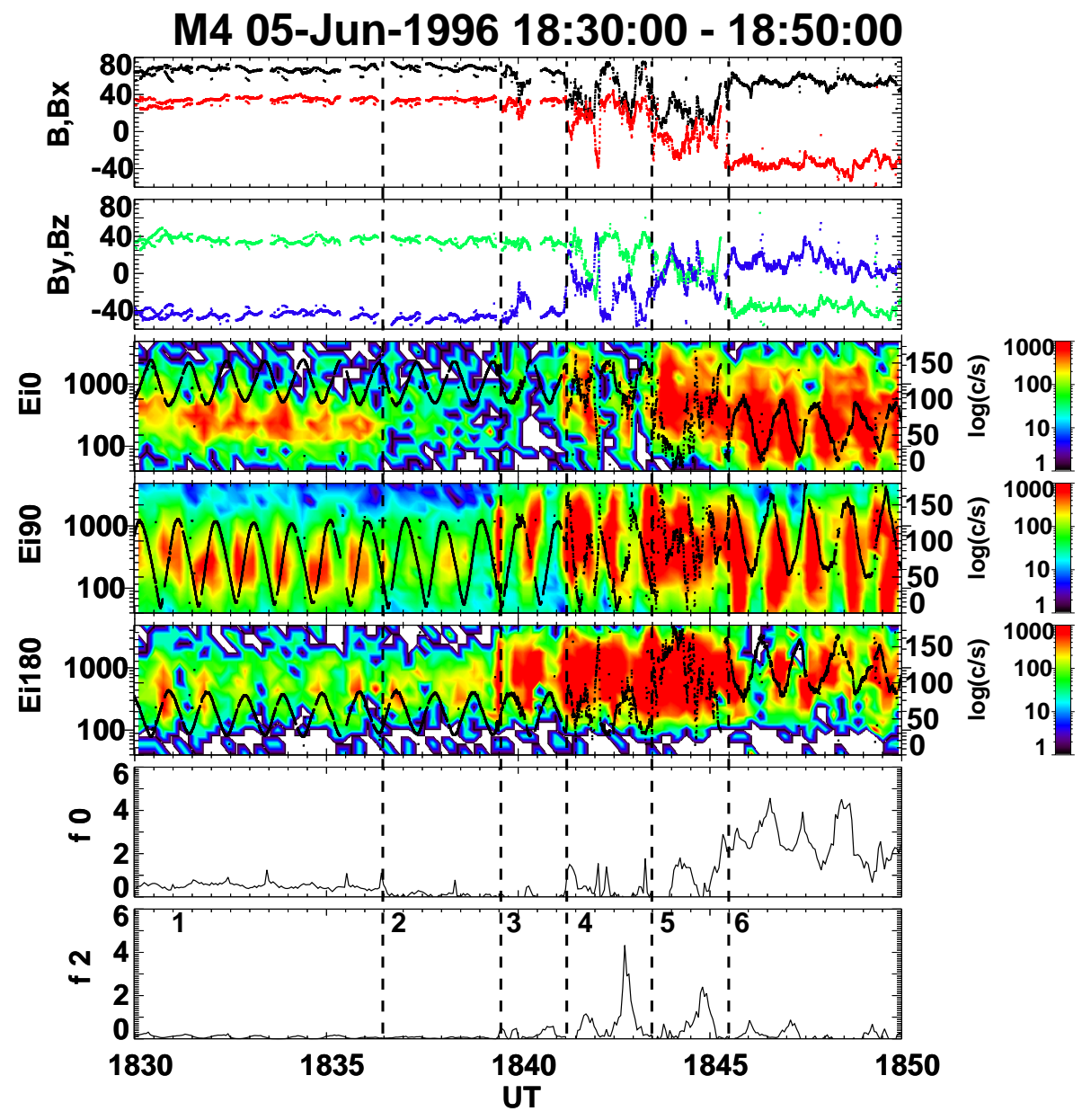

Fig. 4. Vortex-like structure observed by Magion-4. From top to bottom: the strength and the components of the magnetic field (the GSM coordinate system, $B_{x}$ - red, $B_{y}$ - green, $B_{z}$ - blue); spectrograms of tailward (EiO), perpendicular (Ei90), and sunward (Ei180) streaming ions in the energy range from $100 \mathrm{eV}$ to $25 \mathrm{keV}$ together with their pitch angles (black lines inside the spectrograms) and ion fluxes in units of $10^{8} \mathrm{~cm}^{-2} \mathrm{~s}^{-1}$ measured in tailward $(f 0)$ and perpendicular $(f 2)$ directions. The vertical lines and numbers denote the different regions of plasma populations (for their description see to the text).

mantle. These ions entered at the subsolar region during the previous period of southward IMF.

2. The mantle population vanishes because the IMF turned northward and subsolar reconnection was terminated.

3. The changes of the magnetic field and bursts of downgoing ions suggest that the spacecraft entered the outflow region of lobe reconnection proceeding tailward of the spacecraft.

4. The spacecraft crosses the boundary where the magnetic field turns towards the magnetosheath orientation and the direction of the bulk ion motion changes because the ion flux in the perpendicular direction $(f 2)$ becomes much larger than that in the parallel direction $(f 1)$.

5. The plasma is nearly stagnant, the counts in all spectrometer channels are similar and do not depend on the pitch angle. This interval is bounded by a sharp turn of the magnetic field at $\sim 18: 45: 30$ UT.

6. The spacecraft entered the magnetosheath.

Last two panels show the tailward and perpendicular ion fluxes measured by wide-angle Faraday cups. One can see a very low flux in first two intervals because the mantle population is tenuous and slow. The perpendicular flux exhibits two sharp peaks that correspond to crossings of the boundaries of the analyzed structure. The magnetosheath proper can be identified by the increase of the tailward flux.

We would like to stress out that we have found two magnetic field rotations from magnetospheric to magnetosheath orientations - at 18:41 and at 18:45:20 UT, however, only the first of them possesses all attributes of the high-latitude magnetopause. The spacecraft detects a burst of field aligned 

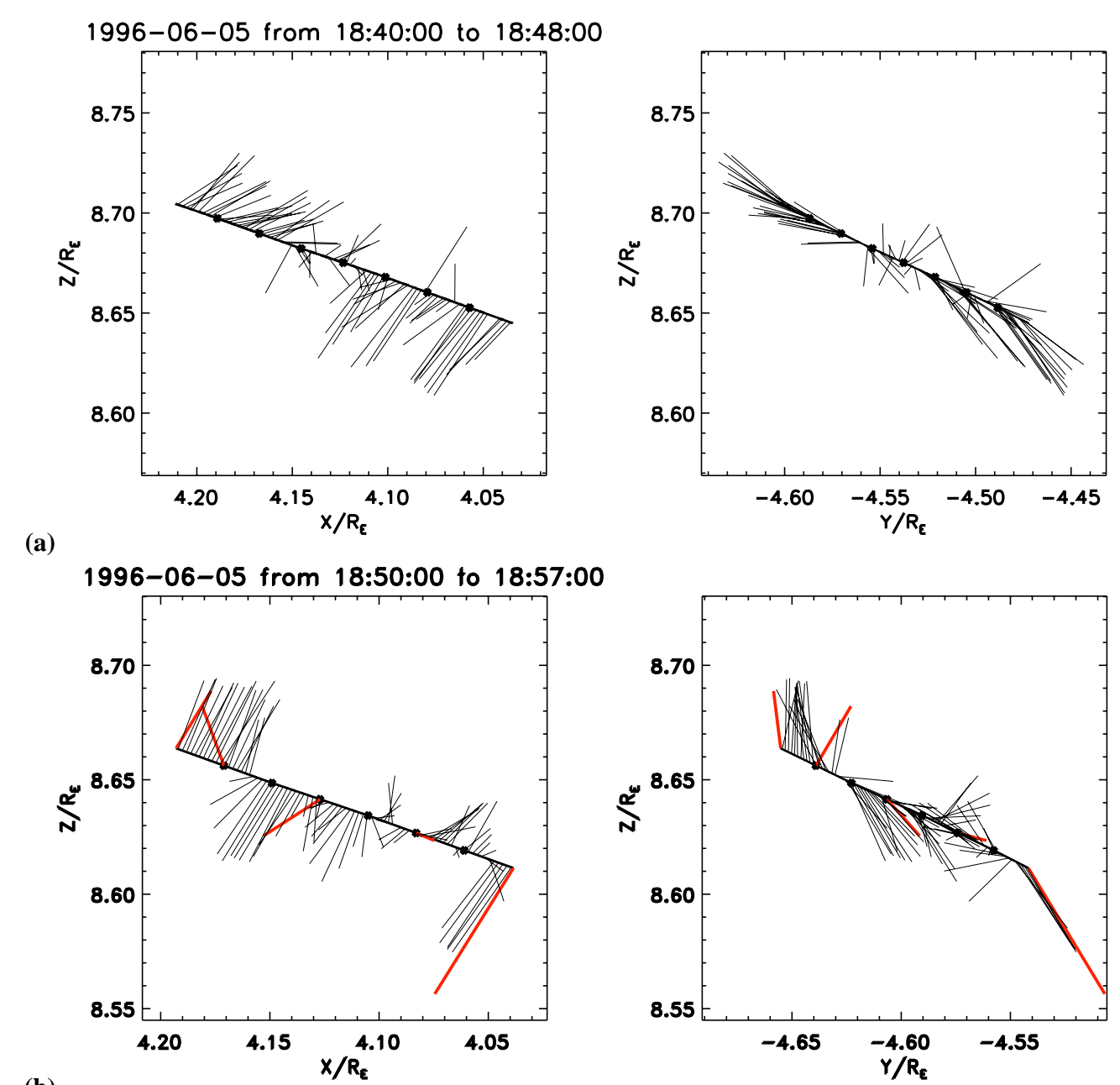

Fig. 5. Projections of magnetic field vectors measured along the orbits of Magion-4 (top) and Interball-1 (bottom). Both spacecraft moved from right to left, the circles on spacecraft trajectories denote the minutes after 18:40 UT (top panels) and after 18:50 UT (bottom panels). The rightmost points denote the time 18:41:00 UT in the top panels and 18:51:00 UT in the bottom panels. In the bottom panel (Interball-1), the red vectors show averaged directions of the ion flow.

ions (spectrogram Ei 90 in Fig. 4) and the ion density significantly increases in course of the first rotation. The second rotation is accompanied with the change of the flow direction but no reconnection outflow was detected. As it can be estimated from the color coding of the spectrograms, the density is similar on both sides of this discontinuity.

A similar structure was registered by Interball-1 about 8 min later, between 18:49:00 and 18:56:30 UT, as it is demonstrated in Fig. 5 where the projections of magnetic field vectors along the spacecraft orbits are shown in time intervals corresponding to the observations of the discussed structure. The spacecraft move from right to left and one can see that both spacecraft observe the magnetospheric orientations at the beginning and, after several rotations, they enter the magnetosheath proper. If we follow the magnetic field changes as observed by Interball-1 (bottom panel in Fig. 5) we can note that the event starts with a sharp turn from a magnetospheric field direction to that resembling the magnetosheath field. A following more or less smooth rotation back toward the magnetospheric orientation is terminated by a new sharp turn to magnetosheath field direction.

Corresponding patterns can be identified in ion flows that are shown by the red arrows in Fig. 5. The time resolution of the ion flow is rather poor but the figure shows that the directions of the ion flow roughly coincide with the magnetic field orientations. It is natural for the rightmost arrow that corresponds to the reconnection jet but a similar alignment can be seen for the rest of the interval that was attributed to the magnetosheath population. Since the ratio of plasma to magnetic pressures, $\beta$, is rather high in this region ( $\sim 80$; plasma parameters are shown in the next figure), we can expect that the magnetic field is frozen into the plasma flow and take the 


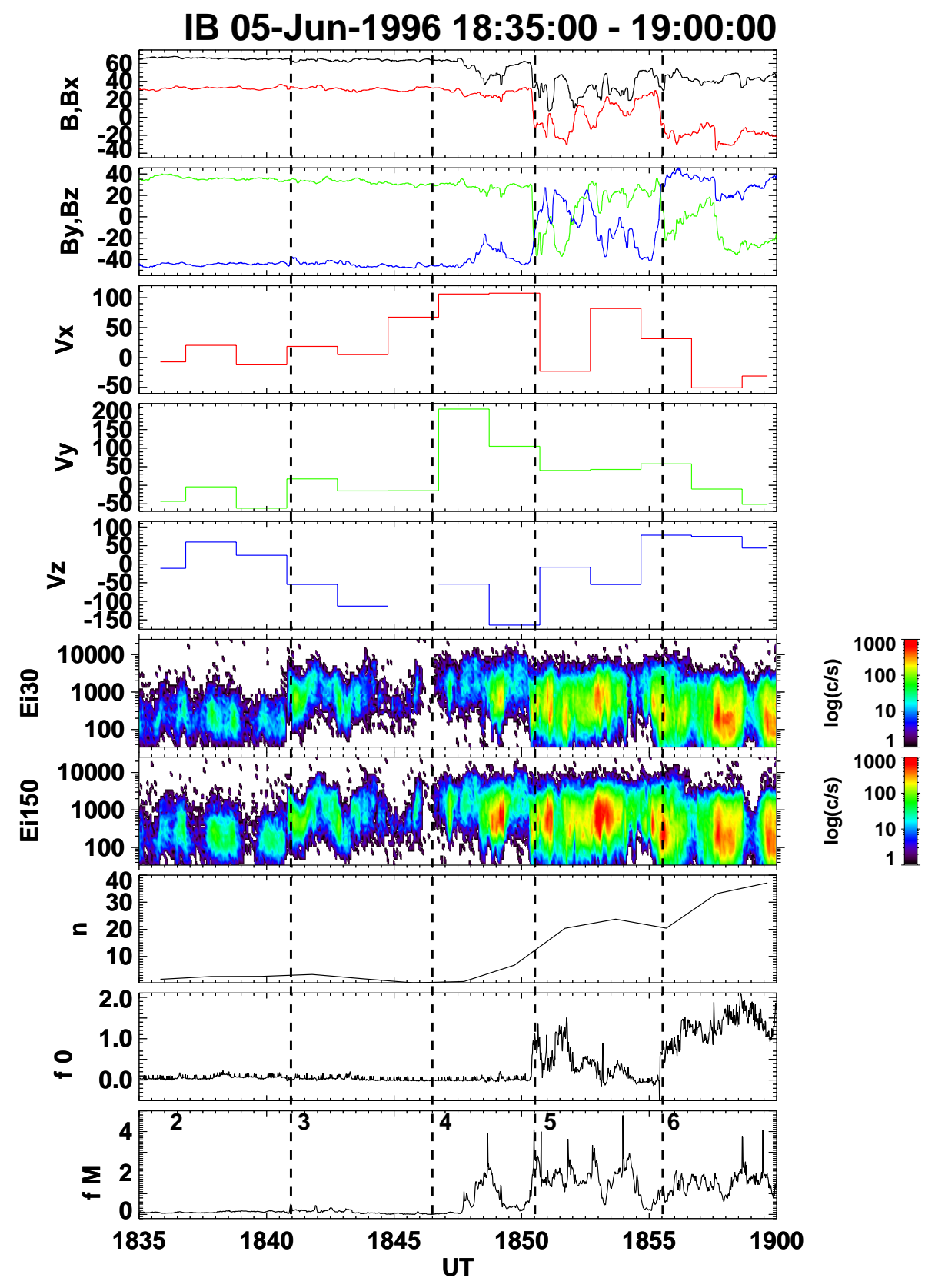

Fig. 6. The same event as in Fig. 4 registered by Interball-1. From top to bottom: the strength and three components of the magnetic field (the GSM coordinate system, $B_{x}$ - red, $B_{y}$ - green, $B_{z}$ - blue); three components of the ion velocity in $\mathrm{km} / \mathrm{s}$; spectrograms of tailward, and sunward streaming ions in the energy range from $100 \mathrm{eV}$ to $25 \mathrm{keV}$; plasma density and ion fluxes in units of $10^{8} \mathrm{~cm}^{-2} \mathrm{~s}^{-1}$ measured in tailward $(f 0)$ and perpendicular $(f M)$ directions. The vertical lines and numbers denote the different regions of plasma populations (for their description see to the text).

magnetic field direction that was measured with a sufficient time resolution as a proxy for direction of the plasma flow.

Here, we should repeat that both satellites moved essentially along the same orbit from the magnetosphere outward and their separation along the orbit was approximately the same as the time lag between observations of the analyzed structure. Thus, we can expect that the whole region was nearly stagnant and we can use the measurements of a more complete plasma spectrometer onboard Interball-1 that are shown in Fig. 6. The organization of the figure is similar to that in Fig. 4 and the vertical lines and numbers of regions have the same meaning. We start with the rest of the 


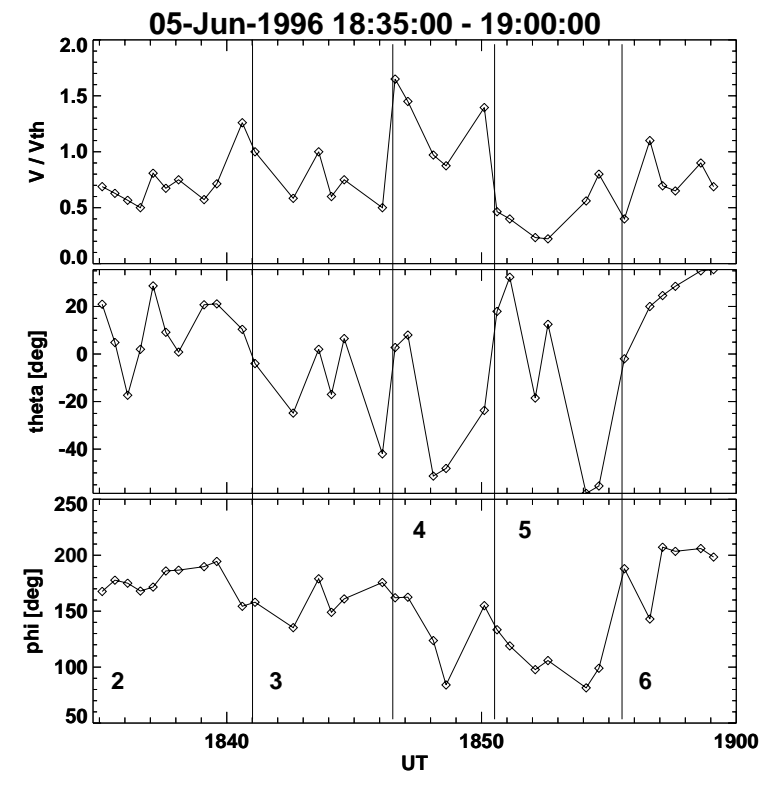

Fig. 7. Plasma flow parameters computed from Interball-1 Faraday cups. From top to bottom: the ratio of bulk-to-thermal velocities, $v / v_{t h}$ and two directional angles: $\theta$ (the angle between the flow vector and the $\mathrm{Z}$-axis), $\phi$ (the angle between the positive $\mathrm{Y}$-direction and the flow vector projection onto the YZ-plane).

mantle population (region 2) that changes to the downstreaming plasma products of lobe reconnection (region 3) that are denser closer to the magnetopause that divides regions 4 and 5. Inside the region 5, the magnetic field as well as the ion velocity smoothly rotate toward the orientation that was observed prior the mentioned magnetopause crossing. This rotation is terminated by a sharp turn of the magnetic field and velocity directions toward those typical for the magnetosheath.

The last two panels show again the ion fluxes measured by Faraday cups in antisunward $(f 0)$ and perpendicular $(f 2)$ directions. These data have a better time resolution and thus allow us to compute the direction of the velocity vector using the procedure developed by Koval et al. (2005). The procedure provides the ratio of bulk-to-thermal velocities $\left(v / v_{t h}\right)$ and two angles of the ion flow direction: $\theta$ and $\phi$. In order to emphasize the changes of the ion flow direction, the theta angle shows the deviation of the ion flow direction from the positive $Z_{\mathrm{GSE}}$ axis (positive values point tailward) and the phi angle is an angle between a projection of the flow vector onto the $Y Z_{\mathrm{GSE}}$ plane and the positive $Y_{\mathrm{GSE}}$ direction. The results of this analysis are depicted in Fig. 7. One can identify the crossing of the magnetopause characterized by an increase of the $v / v_{t h}$ ratio in the region 4 and following smooth rotation of the velocity vector through the region 5 . This rotation is terminated by a sharp turn of the ion flow direction toward the magnetosheath orientation (region 6). We should point out that, although these data show generally the same features as velocity components in Fig. 6, there are differences caused by different measuring methods. The Faraday cups integrate over large spatial angles, and thus they register but they cannot distinguish different components of the plasma flow like reconnection outflows. On the other hand, the electrostatic analyzers scan in time and space and they miss short-living flows that are currently out of their field of view.

As we noted in comments to Fig. 5, the plasma and magnetic field rotate in accord as it can be seen in Fig. 8 where two scans of the ion distribution are plotted in the $v_{\text {par }} / v_{\text {perp }}$ coordinates. The left panel corresponds to the beginning and the right panel to the end of the interval 5. The majority of ions are seen in the direction parallel with the magnetic field $\left(v_{y n}\right)$ in both panels in spite of the fact that both magnetic field and plasma flow changed significantly their directions. We think that it further confirms that the magnetic field is frozen into the plasma flow.

\section{Discussion}

The region 5 observed by Magion- 4 and about eight minutes later by Interball-1 is bounded from both sides by the magnetic field rotation from the magnetospheric to magnetosheath orientation. First of them exhibits principal features of a crossing of the high-latitude magnetopause under northward IMF, i.e. hight magnetic shear and a presence of the ions accelerated by reconnection proceeding tailward. However, the second magnetic field rotation was observed inside a region of a high density and no reconnection outflow was detected. Although lobe reconnection can be bursty (e.g. Šafránková et al., 1998) and thus, reconnection products can absent, we suggest that the second magnetic field and flow rotations cannot be attributed to the magnetopause crossing but that the spacecraft crosses a structure in the magnetosheath and the direction of the magnetic field is determined by a slow plasma motion. The high temperatures inside this region together with the high plasma density cause a diamagnetic effect. Sharp changes of the magnetic field direction on the edges of this structure and a magnetic field rotation inside that can be clearly identified in spite of a high fluctuation level suggest a vortex-like shape of this structure.

Our understanding of a formation of such structure in the cusp region is presented in Fig. 9. This figure repeats Fig. 1 with an addition of the magnetic field line rotating consistently with observations. The figure shows a magnetopause indentation (see also Fig. 2) that creates an obstacle to the plasma flow. We assume that when the fast magnetosheath flow encounters the tailward edge of the magnetopause indentation, an eddy is created and the frozen-in magnetic field lines are rolled up. Since the estimated dimension of the eddy is only about $0.25 R_{E}$, it is oversized in the figure for a better readability. We would like to point out that a similar mechanism was suggested for a plasma penetration through the 

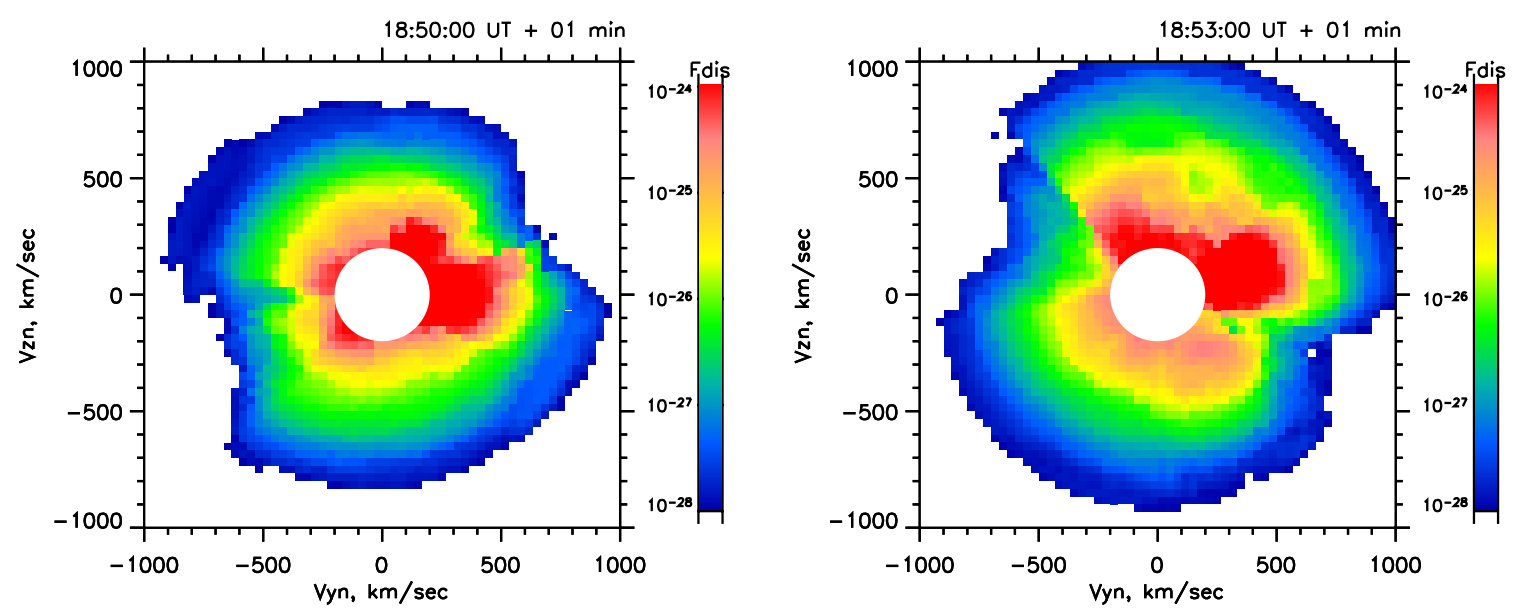

Fig. 8. Ion distribution functions in magnetic field aligned coordinates ( $v_{y n}$ is the component parallel to the magnetic field) for two times. The time of a particular observation is given at the top of each panel.

magnetopause at the flank LLBL (Nykyri et al., 2006) due to the Kelvin-Helmholz instability. The analyzed observations are consistent with crossings of the depicted structure near its tailward edge by Interball- 1 and close to its center by Magion-4. According to this scenario, Interball-1 would observe a reconnection jet directed sunward/southward just prior the exit to the magnetosheath. It is clearly seen in ion spectra and Faraday cup data around $\sim 18: 49$ UT. A fast tailward/northward flow would be seen after the magnetic field rotation. This flow can be identified by a comparison of counts from different analyzers of the ion spectrometer but it cannot be seen in the computed velocity because the full scan of a 3-D distribution needs two minutes. An indication of this flow direction reversal can be seen around $\sim 18: 50$ UT in Fig. 7. Later, Interball-1 would observe a continuous magnetic field and plasma velocity rotations inside the structure (Figs. 5 and 6). New magnetic field and flow direction reversals are observed on the exit from this structure into free-flow magnetosheath ( 18:55 UT; Figs. 5, 6, 7).

A more complicated magnetic field behavior together with nearly zero net ion flux inside the structure would characterize Magion-4 measurements, consistently with the data in Figs. 4 and 5. The observed structure is nearly standing in space because it was observed by two spacecraft with a delay corresponding to their temporal separation along the orbit. Moreover, although the velocity inside the structure is highly variable, the mean velocity computed across the whole structure has only a small $V_{y}$ component that is probably connected with the 3-D nature of the structure and a location of the spacecraft with respect to its center. It allows us to determine the dimension of the structure in the direction of a satellite motion. This width is rather small, about $1800 \mathrm{~km}$, and it can be the reason why it is not observed too often. Taking into account a typical velocity of the magnetopause motion (on average $40 \mathrm{~km} / \mathrm{s}$ according to Dunlop et al., 2005; Es-

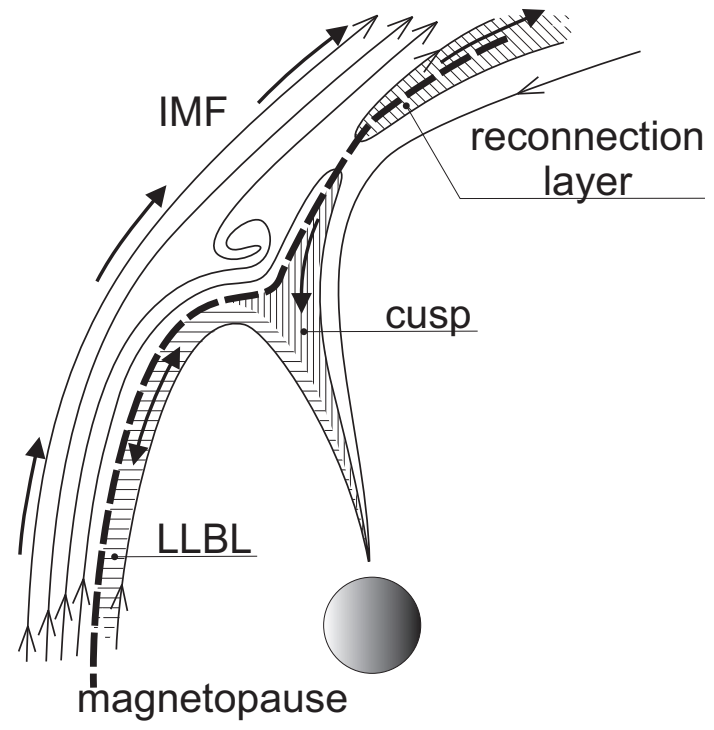

Fig. 9. A sketch of the high-altitude cusp formation under northward IMF with an illustration of a probable shape of the analyzed structure.

coubet et al., 2006), such structure would pass the spacecraft within a minute and it is not clear how such structure would look like when moving together with the magnetopause because its speed is comparable with the speed of the plasma flow within the structure (Fig. 6).

We think that an alternative explanation of our observations by inward and outward motions of the magnetopause within a range of $\approx 1800 \mathrm{~km}$ or by a propagation of a magnetopause surface wave with this amplitude encounters several problems. We observed two sharp rotations of the magnetic field at the edges of the analyzed structure (at 18:50:30 UT 
and 18:55:30 UT, in the Interball-1 data). Both of them are consistent with sharp outward magnetopause crossings and thus, there should be an inward crossing in between them. However, neither magnetic field nor plasma data indicate a presence of such crossing. On the other hand, the rotation of the magnetic field at the leading edge of our structure is accompanied with the reversal of the $V z$ velocity component that is consistent with the lobe magnetopause crossing during northward IMF but only a weak change of the $V z$ component was observed at the trailing edge.

On the other hand, we are using data with a very limited time resolution and, as we pointed above, same important features can be missed. The magnetopause is a layer with a finite thickness and the plasma parameters change almost continuously across it if reconnection in the vicinity of the observing spacecraft is temporarily stopped. Consequently, we cannot exclude that the features observed by both spacecraft can be explained by a periodic "saw-tooth" motion of the magnetopause. In this interpretation, Interball1 crosses the reconnection outflow to the magnetosheath (at $\sim 18: 50$ UT). Then, reconnection is terminated and the spacecraft returns to the magnetosphere due to a slow outward motion of the magnetopause. At $\sim 18: 55 \mathrm{UT}$, the magnetopause suddenly jumps inward and the spacecraft appears in the magnetosheath.

Magion-4 serves as a magnetosheath monitor throughout the analyzed interval. Since it does not observe any significant changes of the magnetic field or plasma parameters that could be attributed to this magnetopause motion an intrinsic oscillation of the outer cup region should be responsible for the observed features.

On the other hand, our interpretation of observations in terms of an eddy caused by the magnetopause indentation can explain "gross scale" features. Fluctuations of all parameters that are superimposed on their averaged profiles suggest that the whole region is in a permanent motion with an amplitude that does not exceed the dimensions of the reported structure.

Based on the presented event, we have carried out a limited statistics of the presence of such structures in the highaltitude cusp region. We have analyzed $\sim 50$ crossings of the magnetopause registered by Magion- 4 and Interball- 1 and found only 4 similar examples. We have made a survey of the 2003 year Cluster magnetic field measurements at the high-latitude magnetopause (Escoubet et al., 2001) and we did not found any similar structure. However, the Cluster orbit is different from that Interball and thus, it is possible that Cluster did not visit the region where such structures are created. Consequently, we cannot make any ultimate conclusion on upstream conditions favorable for creation of such structures. We should stress out that the suggested mechanism is gasdynamic and it is not necessarily associated with reconnection. This feature distinguishes the observed structure from a plasmoid-like structure reported by Zong et al. (2005) that are created during lobe reconnection. On the other hand, we cannot exclude that reconnection can close the magnetic field lines rolled-up by the plasma flow (Fig. 9) but our analysis of the pitch-angle distribution of energetic particles (not shown) suggests rather open than closed field lines.

\section{Conclusion}

We have analyzed one interesting structure in the cuspmagnetosheath interface. We collected all available plasma and magnetic field data connected with this event. Our analysis revealed that the observations are consistent with the presence of a vortex-like structure inside the magnetopause indentation with a diameter $\sim 1800 \mathrm{~km}$. We suggest that this structure is created by the magnetosheath flow that encountered the tailward edge of the magnetopause indentation. The creation of such structures is probably a part of the process that slows down the magnetosheath flow within the cusp indentation. These structures can be observed only during stable upstream conditions because their dimensions are small and they could not be distinguished from an irregular turbulence that is typical for the high-latitude magnetopause around the cusp (e.g. Savin et al., 2001, 2002, 2004) when the whole region is in a permanent motion. However, the time resolution of the available data is poor and thus an alternative explanation in terms of a "saw-tooth" motion of the magnetopause cannot be ruled out.

Acknowledgement. The authors thank A. Fedorov for the Fig. 9 preparation. The present work was partly supported by the Czech Grant Agency under Contracts 205/07/0694 and 202/08/H057, and partly by the Research Plan MSM 0021620860 that is financed by the Ministry of Education of the Czech Republic. O. Tkachenko thanks to the Grant agency of the Charles University for support via project No. 147307.

Topical Editor R. Nakamura thanks C.-P. Escoubet and two other anonymous referees for their help in evaluating this paper.

\section{References}

Alexandrova, O., Mangeney, A., Maksimovic, M., CornilleauWehrlin, N. H., Bosqued, J.-M., and Andre, M.: Alfven vortex filaments observed in magnetosheath downstream of a quasiperpendicular bow shock, J. Geophys. Res., 111(A12), A12208, doi:10.1029/2006JA011934, 2006.

Bogdanova, Y. V., Marchaudon, A., Owen, C. J., Dunlop, M. W., Frey, H. U., Wild, J. A., Fazakerley, A. N., Klecker, B., Davies, J. A., and Milan, S. E.: On the formation of the high-altitude stagnant cusp: Cluster observations, Geophys. Res. Lett., 32, L12101, doi:10.1029/2005GL022813, 2005.

Burch, J. L., Reiff, P. H., Spiro, R. W., Heelis, R. A., and Fields, S. A.: Cusp region particle precipitation and ion convection for northward interplanetary magnetic field, Geophys. Res. Lett., 7, 393-396, 1980.

Burlaga, L. F.: A heliospheric vortex street, J. Geophys. Res., 95, 4333-4336, 1990. 
Cargill, P. J., Dunlop, M. W., Lavraud, B., Elphic, R. C., Holland, D. L., Nykyri, K., Balogh, A., Dandouras, I., and Rème, H.: CLUSTER encounters with the high altitude cusp: boundary structure and magnetic field depletions, Ann. Geophys., 22, 1739-1754, 2004, http://www.ann-geophys.net/22/1739/2004/.

Cargill, P. J., Lavraud, B., Owen, C. J., et al.: Cluster at the magnetospheric cusps, Space Sci. Rev., 118(1-4), 321-366, 2005.

Crooker, N. U.: Dayside merging and cusp geometry, J. Geophys. Res., 84, 951-959, 1979.

Dungey, J. W.: Interplanetary field and auroral zones, Phys. Rev. Lett., 6, 47-49, 1961.

Dungey, J. W.: The structure of the ionosphere, or adventures in velocity space, in: Geophysics: The Earths Environment, edited by: DeWitt, C., Hiebolt, J., and Lebeau, A., 526-536, Gordon and Breach, New York, 1963.

Dunlop, M. W., Balogh, A., and Glassmeier, K.-H.: Fourpoint Cluster application of magnetic field analysis tools: The discontinuity analyzer, J. Geophys. Res., 107(A11), 1385, doi:10.1029/2001JA005089, 2002.

Dunlop, M. W., Lavraud, B., Cargill, P., Taylor, M. G. G. T., Balogh, A., Reme, H., Decreau, P., Glassmeier, K.-H., Elphic, R. C., Bosqued, J.-M., Fazakerley, A. N., Dandouras, I., Escoubet, C. P., Laakso, H., and Marchaudon, A.: Cluster observations of the cusp: Magnetic structure and dynamics, Surv. Geophys., 26(13), 5-55, 2005.

Eastman, T. E., Hones Jr., E. W., Bame, S. J., and Asbridge, J. R.: The magnetospheric boundary layer: Site of plasma, momentum and energy transfer from the magnetosheath into the magnetosphere, Geophys. Res. Lett., 3, 685-688, 1976.

Escoubet, C. P., Fehringer, M., and Goldstein, M.: The Cluster mission, Ann. Geophys., 19, 1197-1200, 2001, http://www.ann-geophys.net/19/1197/2001/.

Escoubet, C. P., Bosqued, J. M., Berchem, J., Trattner, K. J., Taylor, M. G. G. T., Pitout, F., Laakso, H., Masson, A., Dunlop, M., Reme, H., Dandouras, I., and Fazakerley, A.: Geophys. Res. Lett., 33(7), L07108, doi:10.1029/2005GL025598, 2006.

Escoubet, C. P., Berchem, J., Bosqued, J. M., Trattner, K. J., Taylor, M. G. G. T., Pitout, F., Laakso, H., Masson, A., Dunlop, M., Dandouras, I., Reme, H., Fazakerley, A. N., and Daly, P.: Effect of a northward turning of the interplanetary magnetic field on cusp precipitation as observed by Cluster, J. Geophys. Res., 113, A07S13, doi:10.1029/2007JA012771, 2008.

Fuselier, S. A., Petrinec, S. M., and Trattner, K. J.: Stability of the high-latitude reconnection site for steady northward IMF, Geophys. Res. Lett., 27, 473-476, 2000.

Gosling, J. T., Thomsen, M. F., Bame, S. J., Elphic, R. C., and Russell, C. T.: Observations of reconnection of interplanetary and lobe magnetic-field lines at the high-latitude magnetopause, J. Geophys. Res., 96(A8), 14 097-14 106, 1991.

Haerendel, G., Paschmann, G., Sckopke, N., and Rosenbauer, H.: The frontside boundary layer of the magnetosphere and the problem of reconnection, J. Geophys. Res., 83, 3195-3216, 1978.

Hasegawa, H., Fujimoto, M., Phan, T. D., Reme, H., Balogh, A., Dunlop, M. W., Hashimoto, C., and TanDokoro, R.: Transport of solar wind into Earth's magnetosphere through rolled-up KelvinHelmholtz vortices, Nature, 430(7001), 755-758, 2004.

Hasegawa, H., Fujimoto, M., Takagi, K., Saito, Y., Mukai, T., and Reme, H.: Single-spacecraft detection of rolled-up KelvinHelmholtz vortices at the flank magnetopause, J. Geophys. Res.,
111, A09203, doi:10.1029/2006JA011728, 2006.

Hones Jr., E. W., Asbridge, J. R., Bame, S. J., Montgomery, M. D., Singer, S., and Akasofu, S. I.: Measurements of magnetotail plasma flow made with Vela 4B, J. Geophys. Res., 77, 55035522, 1972.

Hones, E. W., Birn, J., Bame, S. J., Asbridge, J. R., Paschmann, G., Sckopke, N., and Haerendel, G.: Further determination of the characteristics of magnetospheric plasma vortices with ISEE 1 and 2, J. Geophys. Res., 86, 814-820, 1981.

Koval, A., Safrankova, J., and Nemecek, Z.: A study of particle flows in hot flow anomalies, Planet Space Sci., 53(1-3), 41-52, 2005.

Kessel, R. L., Chen, S. H., Green, J. L., Fung, S. F., Boardsen, S. A., Tan, L. C., Eastman, T. E., Craven, J. D., and Frank, L. A.: Evidence of high-latitude reconnecting during northward IMF: Hawkeye observations, Geophys. Res. Lett., 23(5), 583586, 1996.

Klimov, S., Romanov, S., Amata, E., et al.: ASPI experiment: Measurements of fields and waves onboard the Interball-1 spacecraft, Ann. Geophys., 15, 514-527, 1997, http://www.ann-geophys.net/15/514/1997/.

Lavraud, B., Dunlop, M. W., Phan, T. D., et al.: Cluster observations of the exterior cusp and its surrounding boundaries under northward IMF, Geophys. Res. Lett., 29(20), 1995, doi:10.1029/2002GL015464, 2002.

Lavraud, B., Fedorov, A., Budnik, E., Grigoriev, A., Cargill, P. J., Dunlop, M. W., Reme, H., Dandouras, I., and Balogh, A.: Cluster survey of the high-altitude cusp properties: a three-year statistical study, Ann. Geophys., 22, 3009-3019, 2004a, http://www.ann-geophys.net/22/3009/2004/.

Lavraud, B., Phan, T. D., Dunlop, M. W., Taylor, M. G. G. T., Cargill, P. J., Bosqued, J.-M., Dandouras, I., Reme, H., Sauvaud, J.-A., Escoubet, C. P., Balogh, A., and Fazakerley, A.: The exterior cusp and its boundary with the magnetosheath: Cluster multi-event analysis, Ann. Geophys., 22, 3039-3054, 2004b, http://www.ann-geophys.net/22/3039/2004/.

Liu, J. and Horton, W.: The intrinsic electromagnetic solitary vortices in magnetized plasma, J. Plasma Phys., 36, 1-24, 1986.

Longmore, M., Schwartz, S. J., Geach, J., Cooling, B. M. A., Dandouras, I., Lucek, E. A., and Fazakerley, A. N.: Dawn-dusk asymmetries and sub-Alfvénic flow in the high and low latitude magnetosheath, Ann. Geophys., 23, 3351-3364, 2005, http://www.ann-geophys.net/23/3351/2005/.

Luhmann, J. R., Walker, R. J., Russell, C. T., Crooker, N. U., Spreiter, J. R., and Stahara, S. S.: Patterns of potential magnetic field merging sites on the dayside magnetopause, J. Geophys. Res., 89, 1741-1744, 1984.

Měrka, J., Šafránková, J., and Němeček, Z.: Cusp-like plasma in high altitudes: A statistical study of the width and location of the cusp from Magion-4, Ann. Geophys., 20, 311-320, 2002, http://www.ann-geophys.net/20/311/2002/.

Měrka, J., Šafránková, J., Němeček, Z., and Šimůnek, J.: Magion-4 high-altitude cusp study, Surv. Geophys., 26(1-3), 57-69, 2005.

Němeček, Z., Fedorov, A., Šafránková, J., and Zastenker, G.: Structure of the low-latitude magnetopause: MAGION-4 observation, Ann. Geophys., 15, 553-561, 1997, http://www.ann-geophys.net/15/553/1997/.

Němeček, Z., Měrka, J., and Šafránková, J.: The tilt angle control of the outer cusp position, Geophys. Res. Lett., 27(1), 77-80, 2000. 
Němeček, Z., Šafránková, J., Přech, L., Šimůnek, J., Sauvaud, J.-A., Stenuit, H., Fedorov, A., Fuselier, S. A., Savin, S., Zelenyi, L., and Berchem, J.: Structure of the outer cusp and sources of the cusp precipitation during intervals of a horizontal IMF, J. Geophys. Res., 108, 1420, doi:10.1029/2003JA009916, 2003.

Němeček, Z., Šafránková, J., Měrka, J., Šimůnek, J., and Přech, L.: Interball contribution to the high-altitude cusp observations, Planet. Space Sci., 55(15), 2286-2294, 2007.

Newell, P. T., Meng, C.-I., Sibeck, D. G., and Lepping, R.: Some low-altitude cusp dependencies on the interplanetary magnetic field, J. Geophys. Res., 94(A7), 8921-8927, 1989.

Nykyri, K. and Otto, A.: Plasma transport at the magnetospheric boundary due to reconnection in KelvinHelmholtz vortices, Geophys. Res. Lett., 28(18), 3565-3568, doi:10.1029/2001GL013239, 2001.

Nykyri, K., Otto, A., Lavraud, B., Mouikis, C., Kistler, L. M., Balogh, A., and Rème, H.: Cluster observations of reconnection due to the Kelvin-Helmholtz instability at the dawnside magnetospheric flank, Ann. Geophys., 24, 2619-2643, 2006, http://www.ann-geophys.net/24/2619/2006/.

Onsager, T. G., Scudder, J. D., Lockwood, M., and Russell, C. T.: Reconnection at the high-latitude magnetopause during northward interplanetary magnetic field conditions, J. Geophys. Res., 106(A11), 25 467-25 488, 2001.

Otto, A. and Fairfield, D. H.: Kelvin-Helmholtz instability at the magnetotail boundary: MHD simulation and comparison with Geotail observations, J. Geophys. Res., 105(A9), 21 175-21 190, 2000.

Palmroth, M., Laasko, H., and Pulkkinen, T. I.: Location of highaltitude cusp during steady solar wind conditions, J. Gephys. Res., 106, 21 109-21 122, 2001.

Panov, E. V., Buchner, J., Franz, M., Korth, A., Savin, S. P., Reme, H., and Fornacon, K.-H.: High-latitude Earth's magnetopause outside the cusp: Cluster observations, J. Geophys. Res., 113(A1), A01220, doi:10.1029/2006JA012123, 2008.

Paschmann, G., Rosenbauer, H., and Sckopke, N.: Heos 2 plasma observations in distant polar cusp, Transactions-American geophysical union, 56(3), p. 170, 1975.

Paschmann, G., Haerendel, G., Sckopke, N., and Rosenbauer, H.: Plasma and magnetic-field characteristics of distant polar cusp near local noon-entry layer, J. Geophys. Res., 81(16), 28832899, 1976.

Petrinec, S. M. and Russell, C. T.: An examination of the effect of dipole tilt angle and cusp regions on the shape of the dayside magnetopause, J. Geophys. Res., 100(A6), 9559-9566, 1995.

Petrinec, S. M. and Russell, C. T.: Near-Earth magnetopause shape and size as determined from the magnetopause flaring angle, J. Geophys. Res., 101, 137-152, 1996.

Rosenbauer, H., Grunwaldt, H., Montgomery, M. D., Paschmann, G., and Sckopke, N.: Heos 2 plasma observations in the distant magnetosphere: The plasma mantle, J. Geophys. Res., 80, 27232737, 1975.

Reiff, P. H., Hill, T. W., and Burch, J. L.: Solar Wind Plasma Injection at the Dayside Magnetospheric Cusp, J. Geophys. Res., 82, 479-491, 1977.

Reiff, P. H.: Evidence of magnetic merging from low-latitude spacecraft and ground-based experiments, in: Magnetic reconnection in Space and Laboratory Plasmas, edited by: Hones Jr., E. W., Geophys. Monogr., AGU, Washington, D.C., 30, 104,
1984.

Russell, C. T., Fedder, J. A., Slinker, S. P., Zhou, X. W., Le, G., Luhmann, J. G., Fenrich, F. R., Chandler, M. O., Moore, T. E., and Fuselier, S. A.: Entry of the POLAR spacecraft into the polar cusp under northward IMF conditions, Geophys. Res. Lett., 25(15), 3015-3018, 1998.

Šafránková, J., Zastenker, G., Němeček, Z., Fedorov, A., Simerský, M., and Přech, L.: Small scale observation of the magnetopause motion: Preliminary results of the INTERBALL project, Ann. Geophys., 15, 562-569, 1997, http://www.ann-geophys.net/15/562/1997/.

Šafránková, J., Němeček, Z., Sibeck, D. G., Přech, L., Měrka, J., and Santolík, O.: Two-point observation of high-latitude reconnection, Geophys. Res. Lett., 25(23), 4301-4304, 1998.

Šafránková, J., Němeček, Z., Dušík, S., Přech, L., Sibeck, D. G., and Borodkova, N. N.: The magnetopause shape and location: A comparison of the INTERBALL and GEOTAIL observations with models, Ann. Geophys., 20, 301-309, 2002a, http://www.ann-geophys.net/20/301/2002/.

Šafránková, J., Měrka, J., and Němeček, Z.: Plasma flow across the cusp-magnetosheath boundary under northward IMF, Adv. Space Res., 30(12), 2787-2792, 2002b.

Šafránková, J., Dušík, S., and Němeček, Z.: The shape and location of the high-latitude magnetopause, Adv. Space Res., 36(10), 1934-1939, doi:10.1016/j.asr.2004.05.009, 2005.

Sandahl, I., Lundin, R., Yamauchi, M., Eklund, U., Šafránková, J., Němeček, Z., Kudela, K., Lepping, R. P., Lin, R. P., Lutsenko, V. N., and Sauvaud, J. A.: Cusp and boundary layer observations by Interball, Adv. Space Res., 20(4-5), 823-832, 1997.

Savin, S. P., Borodkova, N. L., Budnik, E. Yu., et al.: INTERBALL Tail Probe measurements in outer cusp and boundary layers, in: Geospace Mass and Energy Flow: Results from the International Solar-Terrestrial Physics Program, edited by: Horwitz, J. L., Galagher, D. L., and Peterson, W. K., Geophysical Monograph 104, American Geophysical Union, Washington D.C., 2544, 1998.

Savin, S. P., Zelenyi, L. M., Romanov, S. A., et al.: Turbulent boundary layer at the border of geomagnetic trap, JETP Lett., 74(11), 547-551, 2001.

Savin, S., Büchner, J., Consolini, G., Nikutowski, B., Zelenyi, L., Amata, E., Auster, H. U., Blecki, J., Dubinin, E., Fornacon, K. H., Kawano, H., Klimov, S., Marcucci, F., Nemecek, Z., Pedersen, A., Rauch, J. L., Romanov, S., Safrankova, J., Sauvaud, J. A., Skalsky, A., Song, P., and Yermolaev, Yu.: On the properties of turbulent boundary layer over polar cusps, Nonlin. Processes Geophys., 9, 443-451, 2002, http://www.nonlin-processes-geophys.net/9/443/2002/.

Savin, S. P., Zelenyi, L., Romanov, S., et al.: Magnetosheath-cusp interface, Ann. Geophys., 22, 183-212, 2004, http://www.ann-geophys.net/22/183/2004/.

Shukla, P. K., Yu, M. Y., and Stenflo, L.: Electromagnetic drift vortices, Phys. Rev. A, 34, 3478-3480, 1986.

Sibeck, D. G., Paschmann, G., Treumann, R. A., Fuselier, S. A., Lennartsson, W., Lockwood, M., Lundin, R., Ogilvie, K. W., Onsager, T. G., Phan, T.-D., Roth, M., Scholer, M., Sckopke, N., Stasiewicz, K., and Yamauchi, M.: Chapter 5 - Plasma transfer processes at the magnetopause, in: Magnetospheric plasma sources and losses, Space Sci. Rev., 88(1-2), 207-283, doi:10.1023/A:1005255801425, 1999. 
Song, P. and Russell, C. T.: Model of the formation of the lowlatitude boundary layer for strongly northward interplanetary magnetic field, J. Geophys. Res., 97(A2), 1411-1420, 1992.

Sotirelis, T. and Meng, C.-I.: Magnetopause from pressure balance, J. Geophys. Res., 104, 6889-6898, 1999.

Spreiter, J. R. and Briggs, B. R.: Theoretical determination of form of boundary of solar corpuscular stream produced by interaction with magnetic dipole field of earth, J. Geophys. Res., 67(1), 37$51,1962$.

Sundkvist, D., Krasnoselskikh, V., Shukla, P. K., Vaivads, A., Andre, M., Buchert, S., and Reme, H.: In situ multi-satellite detection of coherent vortices as a manifestation of Alfvénic turbulence, Nature, 436(7052), 825-828, doi:1038/nature03931, 2005.

Volwerk, M., Glassmeier, K.-H., Nakamura, R., Takada, T., Baumjohann, W., Klecker, B., Reme, H., Zhang, T. L., Lucek, E., and Carr, C. M.: Flow burst-induced Kelvin-Helmholtz waves in the terrestrial magnetotail, Geophys. Res. Lett., 34(10), L10102, doi:10.1029/2007GL029459, 2007.

Volwerk, M., Zhang, T. L., Glassmeier, K.-H., Runov, A., Baumjohann, W., Balogh, A., Reme, H., Klecker, B., and Carr, C.: Study of waves in the magnetotail region with Cluster and DSP, Adv. Space Res., 41(10), 1593-1597, 2008.
Yermolaev, Yu. I., Fedorov, A. O., Vaisberg, O. L., Balebanov, V. M., Obod, Yu. A., Jimenez, R., Fleites, J., Llera, L., and Omelchenko, A. N.: Ion distribution dynamics near the Earth's bow shock: first measurements with the 2D ion energy spectrometer CORALL on the INTERBALL/Tail-probe satellite, Ann. Geophys., 15, 533-541, 1997, http://www.ann-geophys.net/15/533/1997/.

Zhang, H., Dunlop, M. W., Zong, Q.-G., Fritz, T. A., Balogh, A., and Wang, Y.: Geometry of the high-latitude magnetopause as Observed by Cluster, J. Geophys. Res., 112, A02204, doi:10.1029/2006JA011774, 2007.

Zhou, Y., Matthaeus, W. H., and Dmitruk, P.: Magnetohydrodynamic turbulence and time scales in astrophysical and space plasmas, Rev. Mod. Phys., 76, 1015-1035, 2004.

Zong, Q. G., Fritz, T. A., Spence, H., Zhang, H., Huang, Z. Y., $\mathrm{Pu}, \mathrm{Z}$. Y., Glassmeier, K.-H., Korth, A., Daly, P. W., Balogh, A., and Reme, H.: Plasmoid in the high latitude boundary/cusp region observed by Cluster, Geophys. Res. Lett., 32(1), L01101, doi:10.1029/2004GL020960, 2005. 